\title{
AB INITIO CALCULATIONS OF ELASTIC CONSTANTS OF BCC V-NB SYSTEM AT HIGH PRESSURES
}

A. Landa, J. Klepeis, P. Soderlind, I. Naumov, O. Velikokhatnyi, L. Vitos, A. Ruban

May 4, 2005

Journal of Physics and Chemistry of Solids 
This document was prepared as an account of work sponsored by an agency of the United States Government. Neither the United States Government nor the University of California nor any of their employees, makes any warranty, express or implied, or assumes any legal liability or responsibility for the accuracy, completeness, or usefulness of any information, apparatus, product, or process disclosed, or represents that its use would not infringe privately owned rights. Reference herein to any specific commercial product, process, or service by trade name, trademark, manufacturer, or otherwise, does not necessarily constitute or imply its endorsement, recommendation, or favoring by the United States Government or the University of California. The views and opinions of authors expressed herein do not necessarily state or reflect those of the United States Government or the University of California, and shall not be used for advertising or product endorsement purposes. 
Ab initio calculations of elastic constants of the bec $\mathrm{V}$-Nb system at high pressures

A. Landa ${ }^{\text {a,* }}$,J. Klepeis ${ }^{\text {a }}$, P. Söderlind ${ }^{\text {a }}$, I. Naumov ${ }^{\text {b }}$ O. Velikokhatnyic, L. Vitos ${ }^{\text {d,e }}$, A. Ruban ${ }^{d}$

${ }^{a}$ Physics and Advanced Technologies Directorate, Lawrence Livermore National Laboratory, University of California, P.O. Box 808, Livermore, CA 94550, USA

${ }^{b}$ Department of Physics, University of Arkansas, Fayetteville, AR 72701, USA

${ }^{c}$ Department of Materials Science and Engineering, Carnegie Mellon University, Pittsburgh, PA 15213, USA

${ }^{d}$ Department of Materials Science and Engineering, Royal Institute of Technology, SE10044, Stockholm, Sweden

${ }^{e}$ Research Institute for Solid State Physics and Optics, P.O. Box 49, H-1525 Budapest, Hungary

\begin{abstract}
First-principles total energy calculation based on the exact muffin-tin orbital and full potential linear muffin-tin orbital methods were used to calculate the equation of state and shear elastic constants of bcc $\mathrm{V}, \mathrm{Nb}$, and the $\mathrm{V}_{95} \mathrm{Nb}_{05}$ disordered alloy as a function of pressure up to $6 \mathrm{Mbar}$. We found a mechanical instability in $C_{44}$ and a corresponding softening in $C^{\prime}$ at pressures $\sim 2$ Mbar for $\mathrm{V}$. Both shear elastic constants show softening at pressures $\sim 0.5 \mathrm{Mbar}$ for $\mathrm{Nb}$. Substitution of 5 at. $\%$ of $\mathrm{V}$ with $\mathrm{Nb}$ removes the instability of $\mathrm{V}$ with respect to trigonal distortions in the vicinity of 2 Mbar pressure, but
\end{abstract}

\footnotetext{
* Corresponding author. E-mail address: landa1@1lnl.gov (A. Landa). Tel.: +1-925-424-3523.
} 
still leaves the softening of $C_{44}$ in this pressure region. We argue that the pressure induced shear instability (softening) of $\mathrm{V}(\mathrm{Nb})$ originates from the electronic system and can be explained by a combination of the Fermi surface nesting, electronic topological transition, and band Jahn-Teller effect.

Keywords: A. Metals, D. Elastic properties, D. Electronic structure, D Fermi surface.

\section{Introduction}

The group- $\mathrm{VB}$ transition metals $(\mathrm{V}, \mathrm{Nb}$, and $\mathrm{Ta})$ have been subject of numerous experimental and theoretical studies [1-15] in $60 \mathrm{~s}-80 \mathrm{~s}$ due to the high superconducting transition temperatures of its components (for example, $\mathrm{Nb}$ has the highest superconducting transition temperature $T_{c}=9.25 \mathrm{~K}$ among the elemental metals) and unique mechanical properties at extremely high temperatures that place these metals in the position of basic building blocks of intermetallic compounds. However at that time, most of this research was restricted to ambient pressure conditions or pressures bellow 50 kbar. During the past 15 years the development of new techniques for electric and magnetic measurements in a diamond-anvil cell (DAC) has allowed for performing measurements at a megabar pressures. Struzhkin et al [16] measured the superconducting transition temperature of $\mathrm{Nb}$ in the pressure range up to $1.32 \mathrm{Mbar}$. They observed anomalies in $T_{c}(p)$ at 50-60 kbar and 600-700 kbar and suggested that these anomalies arise from electronic topological transitions (ETT). Later Ishizuka et al [17] performed similar DAC experiments on $\mathrm{V}$ in the pressure range up to $1.2 \mathrm{Mbar}$. They discovered that $T_{c}=5.3 \mathrm{~K}$ at ambient pressure and increases linearly with pressure and reaches 17.2 
$\mathrm{K}$ (the highest $T_{c}$ among the elemental metals reported so far) at 1.2 Mbar. However, in contrast to $\mathrm{Nb}$, these measurements did not find any indications of anomalies in $T_{c}$ for $\mathrm{V}$ within this pressure range. Takemura [18] performed high-pressure DAC powder X-ray diffraction experiments on $\mathrm{V}$ and $\mathrm{Nb}$ and found no anomalies, which could be connected with ETT, in the equations of state (EOS) up to the maximum pressure of $1.54 \mathrm{Mbar}$. In order to explore the possibility of a structural phase transition from bcc to another phase, Suzuki and Otani [19] performed the first-principles calculations of lattice dynamics of V in the pressure range up to 1.5 Mbar. They found that the transverse acoustic phonon mode TA $[\xi 00]$ around $\xi=1 / 4$ shows a dramatic softening under pressure and becomes imaginary at pressures higher than $\sim 1.3$ Mbar, indicating a possibility of such structural phase transition. Since in the limit of long-wave lengths $(q \rightarrow 0)$ this mode is directly related to the elastic constant $C_{44}$, clear understanding of the anomaly in the TA curve is principally important in the problem of the shear lattice stability of bcc V. The authors, however, did not discuss the physical reasons behind the dip in the TA.

This paper is devoted to the $a b$ initio study of the EOS and shear elastic constants of $\mathrm{V}, \mathrm{Nb}$, and the $\mathrm{V}_{95} \mathrm{Nb}_{05}$ disordered alloy in the pressure range up to $6 \mathrm{Mbar}$. We used selected density functional theory (DFT) approaches to model the V-Nb system at ambient pressure as well as extreme conditions. We discuss the physical nature of the anomalies in the shear elastic constants of V in terms of the combined Fermi surface (FS) nesting, ETT, and band Jahn-Teller (J-T) effect.

The paper is organized as follows. Pertinent details of computation methods are described in Section 2, followed by results Section 3 and discussion Section 4. We present our conclusions in Section 5. 


\section{Computational details}

In the present paper we employ two complimentary computational techniques. The calculations we have referred to as exact muffin-tin orbitals (EMTO) are performed using a scalar-relativistic Green's function technique based on an improved screened KorringaKohn-Rostoker method, where the one-electron potential is represented by optimized overlapping muffin-tin (OOMT) potential spheres [20-24]. Inside the potential spheres the potential is spherically symmetric and it is constant between spheres. The radii of the potential spheres, the spherical potentials inside the spheres, and the constant value from the interstitial are determined by minimizing (i) the deviation between the exact and overlapping potentials, and (ii) the errors coming from the overlap between spheres. Thus, the OOMT potential ensures a more accurate description of the full potential compared to the conventional muffin-tin or non-overlapping approach.

Within the EMTO formalism, the one-electron states are calculated exactly for the OOMT potentials. As an output of the EMTO calculations, one can determine the selfconsistent Green's function of the system and the complete, non-spherically symmetric charge density. Finally, the total energy is calculated using the full charge density technique $[23,25]$. For the exchange/correlation approximation we use the generalized gradient approximation (GGA) [26]. For the total energy of random substitutional alloys, the EMTO method has been recently combined with the coherent potential approximation (CPA) [24, 27].

The calculations are performed for a basis set including valence spd orbitals whereas the core states were recalculated at each iteration. Integration over the 
irreducible wedge of the Brillouin zone (IBZ) is performed using the special $k$-point method [28] with $819 k$-points for bcc lattice. The Green's function has been calculated for 60 complex energy points distributed exponentially on a semicircle with a $3.5 \mathrm{Ry}$ ( 3.8 Ry for $\mathrm{V}_{95} \mathrm{Nb}_{05}$ alloy) diameter enclosing the occupied states. The equilibrium density is obtained from a Murnaghan fit [29] to about 16 total energies calculated as a function of the lattice constant. For the calculation of $C^{\prime}$ and $C_{44}$ we use volume conserving orthorhombic and monoclinic setups for tetragonal and trigonal distortions, respectively [30], performing integration over the IBZ with $15625(C)$ and $17457\left(C_{44}\right) k$-points.

As EMTO method does not allow us to calculate the energy bands, we applied a fullpotential linear muffin-tin orbitals (FPLMTO) method [31-33] for this purpose. This latter method has no geometrical approximations to its one-electron potential or charge density and is therefore well suited for low symmetry or distorted crystals (we also used this method for shear elastic constants calculations for $\mathrm{V}$ and $\mathrm{Nb}$ ). Within FPLMTO approach, the crystal is divided up into regions inside atomic spheres, where Schrödinger's equation is solved numerically, and an interstitial region. In all LMTO methods the wavefunctions in the interstitial region are Hankel functions. An interpolation procedure is used for evaluating interstitial integrals involving products of Hankel functions. The triple- $k$ basis is composed of three sets of $s, p, d$, and $f$ LMTOs per atom with Hankel function kinetic energies of $-k^{2}=-0.01,-1.0$, and -2.3 Ry (48 orbitals per atom). The Hankel functions decay exponentially as $e^{-k r}$. The angular momentum sums involved in the interpolation procedure are carried up to a maximum of $l=6$. For the exchange/correlation approximation we again use the GGA [26]. The integration over 
the IBZ was carried out using the tetrahedron method [34] with $48 \times 48 \times 48$ shifted mesh resulting in 14724 and $14700 k$-points for $\mathrm{C}^{\prime}$ and $\mathrm{C}_{44}$ shear elastic constants, respectively.

The generalized susceptibility of non-interacting electrons was selected in order to detect the nesting at the FS. This function was calculated by using the standard linear muffin-tin orbitals method in the atomic sphere approximation with so-called combined correction terms [35] and the highly precise analytic tetrahedron method [36]: in order to reach the high precision, the IBZ of the bcc lattice was divided into 3375 tetrahedra.

\section{Results}

Figure 1 shows the EOS (EMTO) for $\mathrm{V}$ and $\mathrm{Nb}$ as well as for the $\mathrm{V}_{95} \mathrm{Nb}_{05}$ disordered alloy. For $\mathrm{V}$, calculated equilibrium volume $\left(V_{0}\right)$ and bulk modulus $\left(B_{0}\right)$ are $13.65 \AA^{3}$ and $1.832 \mathrm{Mbar}$, respectively. FPLMTO calculations give $V_{0}=13.53 \AA^{3}$ and $B_{0}=1.827$ Mbar. These results are in good agreement with experimental density of $\mathrm{V}, V_{0}=13.91 \AA^{3}$ [37], and recently measured bulk modulus, $B_{0}=1.880 \mathrm{Mbar}$ [18], as well as results of FPLMTO calculations by Suzuki and Otani, $B_{0}=1.946$ Mbar [19]. For Nb, EMTO calculations give $V_{0}=18.41 \AA^{3}$ and $B_{0}=1.666 \mathrm{Mbar}$, and FPLMTO calculations give $V_{0}$ $=18.26 \AA^{3}$ and $B_{0}=1.707$ Mbar. Both EMTO and FPLMTO results are in good

agreement with experimental density of $\mathrm{Nb}, V_{0}=17.97 \AA^{3}$ [37], and recently measured bulk modulus, $B_{0}=1.680$ Mbar [18].

Figure 2 and Figure 3 show pressure dependence of the shear elastic moduli of $\mathrm{V}$ and $\mathrm{Nb}$, respectively. EMTO calculations reveal a mechanical instability in $C_{44}$ and corresponding softening in $C^{\prime}$ at pressures $\sim 2$ Mbar for $\mathrm{V}$ (similar tendency is observed from FPLMTO calculations, but at a lower pressure). Both shear elastic constants show 
softening at pressures $\sim 0.5 \mathrm{Mbar}$ for $\mathrm{Nb}$. One should also mention that similar softening for $C_{44}$ modulus, although less pronounced, was predicted from FPLMTO calculations for $\mathrm{Ta}$ [39], the remaining representative of the group-VB. In order to correlate these phenomena with the electronic structure behavior we constructed (FPLMTO) band energies for $\mathrm{V}$ (undistorted and under the trigonal distortion) at different pressures (Figure 4 - Figure 6). As one can see from Figure 4 (experimental equilibrium volume), the trigonal distortion causes the levels with $t_{2 g}$ symmetry $\left(\Gamma_{25^{\prime}}\right.$ and $\left.H_{25}\right)$ to split and levels $\Delta_{2}$ and $\Sigma_{3}$ to hybridize with levels $\Delta_{5}$ and $\Sigma_{1}$, respectively. Similar features of the band energies of V were also described by Ohta and Shimizu [15]. The 3-fold degenerate $\Gamma_{25^{\circ}}$ pure $d$-state is unoccupied at low pressure but shifts to lower energy and passes through the Fermi level as pressure increases (Figure 5 and Figure 6).

Consider now how the density of states (DOS) behaves under pressure and distortion. Figure $7 \mathrm{a}$ shows the increment due to the trigonal distortion of the DOS at the Fermi level, $\Delta N\left(E_{F}\right)$, as a function of pressure. This plot shows that for $\mathrm{V}, \Delta N\left(E_{F}\right)$ increases under deformations at ambient pressure but begins to decrease as pressure increases and passes a minimum in the vicinity of $\sim 3$ Mbar. Additional analysis indicates that as the value of the deformation decreases $(\delta \rightarrow 0), \Delta N\left(E_{F}\right)$ the minimum moves right approaching the region where the $\Gamma_{25}$ point crosses the Fermi level $(\sim 3.3 \mathrm{Mbar}$ according to EMTO calculations). Further compression causes $\Delta N\left(E_{F}\right)$ to increase again monotonically. On the other hand, the Madelung (electrostatic) contribution to the $C_{44}$ elastic constant is positive for all pressures under consideration (Figure 7b).

The pressure evolution of the FS of V can be understood from its cross sections in the central $\{100\}$ (triangular $(\Gamma H N)$ ) and $\{110\}$ (rectangular $(\Gamma H N P N)$ ) planes shown in 
Figure 8a (the notations are borrowed from Mackintosh and Andersen [40]). Since V contains 5 valence electrons per atom, they fill entirely the first, most of the second and a significant part of the third conduction band. In this figure, the first filled band is not shown, the second band (2) has a closed hole-surface centered at the point $\Gamma$ and has the shape of a distorted octahedron. The third band (3) has closed distorted hole-ellipsoids centered at points $N$ and multiple connected opened hole-tubes, so called 'jungle-gym' (JG), which extend from $\Gamma$ to $H$ in the [100] $(\Delta)$ directions. At ambient pressure, the calculated FS sections for V look similar to previously reported theoretical results $[1,3$, $5,7,9,11-14]$, however, at elevated pressures we did not find any evidences of the ETT predicted by Papaconstantopoulos et al [5] whose calculations revealed that at the 5\% reduced lattice spacing, which corresponds to $\sim 240$ kbar pressure, the distorted hole ellipsoids around points $N$ merge with the J-G hole tubes creating a such kind of a hole neck along the $\Gamma-N(\Sigma)$ direction. Instead we found that as pressure increases, the distorted octahedron hole-pocket around the $\Gamma$ point shrinks, indicating a movement of this point towards the Fermi level (Figure 8b), and the J-G hole tube terminates at the [100] direction (Figure 8c).

Calculated at ambient pressure generalized susceptibility, $\chi(q)$, along the $\Gamma-H([\xi 00])$ direction (Figure 9a) shows a slightly pronounced peak at $\xi \approx 0.24$. Detailed analysis of partial contributions to the generalized electron susceptibility indicates that this peak is due to $3^{\text {rd }} \rightarrow 3^{\text {rd }}$ electron transitions (Figure $9 \mathrm{~b}$ ) or, in other words, due to the nesting properties of the FS in the $3^{\text {rd }}$ band (intra-band nesting). As can be seen from Figure 9b, this peak shifts swiftly towards the smaller $\xi$ (lower wave vector $q$ ) as pressure is applied, for example, at 1.8 Mbar the peak is located at $\xi \approx 0.06$. The half of the nesting 
vector $\left(\left|\boldsymbol{q}_{\boldsymbol{n}}\right| / 2 \approx \pi / a[0.24,0,0]\right)$ can be clearly seen in Figure $8 \mathrm{a}$. It corresponds to the position of the anomaly in the TA found by Suzuki and Otani [19]. As pressure increases, $\left|\boldsymbol{q}_{\boldsymbol{n}}\right|$ decreases (Figure 8b) and finally becomes equal to zero at pressure of the above mentioned termination of the J-G tube.

Figure 10 and Figure 11 show calculated (EMTO) pressure dependence of the $C_{44}$ elastic modulus and $\Delta N\left(E_{F}\right)$ of the $\mathrm{V}_{95} \mathrm{Nb}_{05}$ disordered alloy, respectively. Notice that an addition of only 5 at. $\%$ of $\mathrm{Nb}$ stabilizes $\mathrm{V}$ mechanically, whereas significant softening in the $C_{44}$ shear modulus still takes place at $\sim 2$ Mbar pressure. This feature correlates with $\Delta N\left(E_{F}\right)$ behavior - it decreases as pressure increases up to $\sim 3 \mathrm{Mbar}$, but for the $\mathrm{V}_{95} \mathrm{Nb}_{05}$ disordered alloy this effect is significantly smaller than for pure $\mathrm{V}$.

\section{Discussion}

From the data presented above it is clear that softening in the shear constants of $\mathrm{V}$ and $\mathrm{Nb}$ is entirely due to the band structure features and not to the electrostatic forces (the latter stabilize the lattice under pressure). This softening can be attributed simultaneously to three different electronic structure peculiarities, namely, to the FS nesting, ETT, and the band $\mathrm{J}-\mathrm{T}$ effect.

The nesting vector $\boldsymbol{q}_{n}$, spanning two flat pieces of the FS in the $3^{\text {rd }}$ band, already exists at zero pressure and leads to the Kohn anomaly in the transverse acoustic phonon mode TA [ $\xi 00]$ [19] for small $\left|\boldsymbol{q}_{n}\right| / 2 \approx 0.24 \pi / a$. This anomaly also softens the elastic constant $C_{44}$ because in the limit of long waves $(q \rightarrow 0) \rho \omega^{2}(q) / q^{2} \rightarrow C_{44}$. It is remarkable that under pressure $\boldsymbol{q}_{n}$ shrinks at a fast rate and the effect of the Kohn anomaly on $C_{44}$ increases. As soon as $\boldsymbol{q}_{n}$ turns to zero, the ETT, when the neck between two electronic 
sheets of the FS appears (the J-G hole-tube terminates at the [100] direction), takes place. Qualitatively, it is analogous to the event when the FS touches the BZ boundary and is always accompanied by a sharp minimum in the shear elastic constants [41]. So as $\boldsymbol{q}_{n} \rightarrow$ 0 , the softening in $C_{44}$ develops up to the point when $\boldsymbol{q}_{n}$ turns to zero. It is very important that the condition $\boldsymbol{q}_{n}=0$ is reached long before the Fermi level passes the tripledegenerate term $\Gamma_{25}$.

The pressure-induced shear instability in $\mathrm{V}$ can be also, partially, due to the "band J-T effect'. As already was mentioned, the trigonal distortion splits the terms $\Gamma_{25^{\prime}}$ and $H_{25^{\prime}}$ and, besides, leads to a hybridization between $\Delta_{2}$ and $\Delta_{5}$ from one side and between $\Sigma_{3}$ and $\Sigma_{1}-$ from the other. All these splitting/shifting/hybridization effects tend to open the energy gaps on the Fermi level and decrease the total energy. For example, the initially triple-degenerate $\Gamma_{25^{\prime}}$ point splits in such a way that one of the split level goes up above and another two go down below the Fermi level causing the DOS at the Fermi level to decrease.

\section{Conclusions}

First-principles total-energy calculations reveal a tendency for the group-VB transition metals $\mathrm{V}$ and $\mathrm{Nb}$ to soften their shear elastic moduli at elevated pressures. We conclude that this pressure-induced softening is due to the electronic system, exclusively, and has a complicated mechanism. For $\mathrm{V}$, expansion of the pressure range to higher values (above 1.5 Mbar) is desirable to obtain information on possibility of a structural transition from bcc to another phase. The proposed study should be a real challenge for experimentalists because these measurements must be performed at a maximum low 
temperature (softening of the shear elastic constants disappears as temperature increases) and the hydrogen should be removed from the samples (there is a tendency of the elastic constant $C_{44}$ of $\mathrm{V}$ and $\mathrm{Nb}$ to increase with increasing hydrogen concentration [42]).

\section{Acknowledgements}

A.L. would like to thanks D.A. Papaconstantopoulos for helpful discussion. This work was performed under the auspices of the US Department of Energy by the University of California Lawrence Livermore National Laboratory under contract W7405-Eng-48. I.N is grateful to the Office of Naval Research, Grant No. 00014-03-10598. L.V and A.R. are grateful to the Swedish Research Council, The Swedish Foundation for Strategic Research, and the Royal Swedish Academy of Sciences. L.V. also acknowledges the research projects OTKA T046773 and T048827 of the Hungarian Scientific Research Fund.

\section{References:}

1. J.R. Anderson, J.W. McCaffrey, and D.A. Papaconstantopoulos, Solid St. Comm. 7 (1969) 1439.

2. L.F. Mattheiss, Phys. Rev. B 1 (1970) 373.

3. M. Yasui, E. Hayashi, and M. Shimizu, J. Phys. Soc. Jap. 29 (1970) 1446.

4. M.H. Halloran, J.H. Condon, J.E. Graebner, J.E. Kunzler, and F.S.L. Hsu, Phys. Rev. B 1 (1970) 366.

5. D.A. Papaconstantopoulos, J.R. Anderson, and J.W. McCaffrey, Phys. Rev. B 5 (1972) 1214. 
6. J.R. Anderson, D.A. Papaconstantopoulos, J.W. McCaffrey, and J.E. Schirber, Phys. Rev. B 7 (1973) 5115.

7. S. Wakoh and J. Yamashita, J. Phys. Soc. Jap. 35 (1973) 1394.

8. R.D. Parker and M.H. Halloran, Phys. Rev. B 9 (1974) 4130.

9. S. Wakoh, Y. Kubo, and J. Yamashita, J. Phys. Soc. Jap. 38 (1975) 416.

10. N. Elyashar and D.D. Koelling, Phys. Rev. B 15 (1977) 3620.

11. L.L. Boyer, D.A. Papaconstantopoulos, and B.M. Klein, Phys. Rev. B 15 (1977) 3685.

12. D.G. Laurent, C.S. Wang, and J. Callaway, Phys. Rev. B 17 (1978) 455.

13. D.A. Papaconstantopoulos and B.M. Klein, Physica 107 B (1981) 725.

14. J.R. Anderson, D.A. Papaconstantopoulos, and J.E. Schirber, Phys. Rev. B 24 (1981) 6790.

15. Y. Ohta and M. Shimizu, J. Phys. F: Met. Phys. 13 (1983) 761.

16. V. V. Struzhkin, Y. A. Timofeev, R.J. Hemley, and H.-K. Mao, Phys. Rev. Lett. 79 (1997) 4262.

17. M. Ishizuka, M. Iketani, and S. Endo, Phys. Rev. B 61 (2000) R3823.

18. K. Takemura, in M.H. Manghnani, W.J. Nellis, and M.F. Nicol (Eds.), Proceedings of the International Conference on High Pressure Science and Technology (AIRAPT17), Honolulu, HA, 1999, pp. 443-444.

19. N. Suzuki and M. Otani, J. Phys.: Condens. Matter 14 (2002) 10869.

20. O.K. Andersen, O. Jepsen, and G. Kreir, in: V. Kumar, O.K. Andersen, and A. Mookerjee (Eds.), Methods of Electronic Structure Calculations, World Scientific, Singapore, 1994, pp. 63-124. 
21. O.K. Andersen, C. Arcangeli, R.W. Tank, T. Saha-Dasgupta, G. Kreir, O. Jepsen, and I. Dasgupta, in: P.E.A Turchi, A. Gonis, and L. Colombo (Eds.), Tight-Binding Approach to Computational Materials Science, M.R.S. Symposia Proceedings No 491, Materials Research Society, Warrendale, 1998, pp. 3-34.

22. L. Vitos, H.L. Skriver, B. Johansson, and J. Kollár, Comp. Mater. Sci. 18 (2000) 24.

23. L. Vitos, Phys. Rev. B 64 (2001) 014107.

24. L. Vitos, in: Recent Res. Devel. Physics, 5 (2004), Transworld Research Network Publisher, Trivandrum, pp. 103-140.

25. J. Kollár, L. Vitos, and H. L. Skriver, in H. Dreyssé (Ed.), Electronic Structure and Physical Properties of Solids: The Uses of the LMTO Method, Lecture Notes in Physics, Springer, Berlin, 2000, pp. 85-113.

26. J.P. Perdew, K. Burke, and M. Ernzerhof, Phys. Rev. Lett. 77 (1996) 3865.

27. L. Vitos, I.A. Abrikosov, and B. Johansson, Phys. Rev. Lett. 87 (2001) 156401.

28. D. J. Chadi and M.L. Cohen, Phys. Rev. B 8 (1973) 5747; S. Froyen, ibid. 39 (1989) 3168.

29. F.D. Murnaghan, Proc. Natl. Acad. Sci. USA 30 (1944) 244.

30. M.J. Mehl, B.M. Klein, and D.A. Papaconstantopoulos, in: J.H. Westbrook and R.L. Fleischer (Eds.), Intermetallic Compounds: Principles and Practice: Vol. 1 Principles, John Wiley and Sons, Inc., London, 1995, Chap. 9, pp. 195-210.

31. M. Methfessel, Phys. Rev. B 38 (1988) 1537.

32. M. Methfessel, C.O. Rodriguez, and O.K. Andersen, Phys. Rev. B 40 (1989) 2009. 
33. M. Methfessel, M. van Schilfgaarde, and R.A. Casali, in: H. Dreyssé (Ed.), Electronic Structure and Physical Properties of Solids: The Uses of the LMTO Method, Lecture Notes in Physics, Springer, Berlin, 2000, pp. 114-147.

34. P.E. Blöchl, O. Jepsen, and O.K. Andersen, Phys. Rev. B 49 (1994) 16223.

35. H.L. Skriver, The LMTO Method, Springer, Berlin, 1984, 281 p.

36. J. Rath and A.J. Freeman, Phys. Rev. B 11 (1975) 2109.

37. C. Kittel, Introduction to Solid State Physics, John Wiley and Sons, Inc., New York, 1986, 648 p.

38. N. W. Ashcroft and N.D. Mermin, Solid State Physics, Holt, Rinehart, and Winston, New York, 1976, 826 p.

39. P. Söderlind and J. Moriarty, Phys. Rev. B 57 (1998) 10340.

40. A.R. Mackintosh and O.K. Andersen, in: M. Springford (Ed.), Electrons at the Fermi Surface, Cambridge University Press, Cambridge, 1980, pp. 149-224.

41. M.I. Katsnelson, I.I. Naumov, and A.V. Trefilov, Phase Transitions 49 (1994) 143.

42. E.S. Fisher and J.F. Remark, J. Appl. Phys. 51 (1980) 927. 


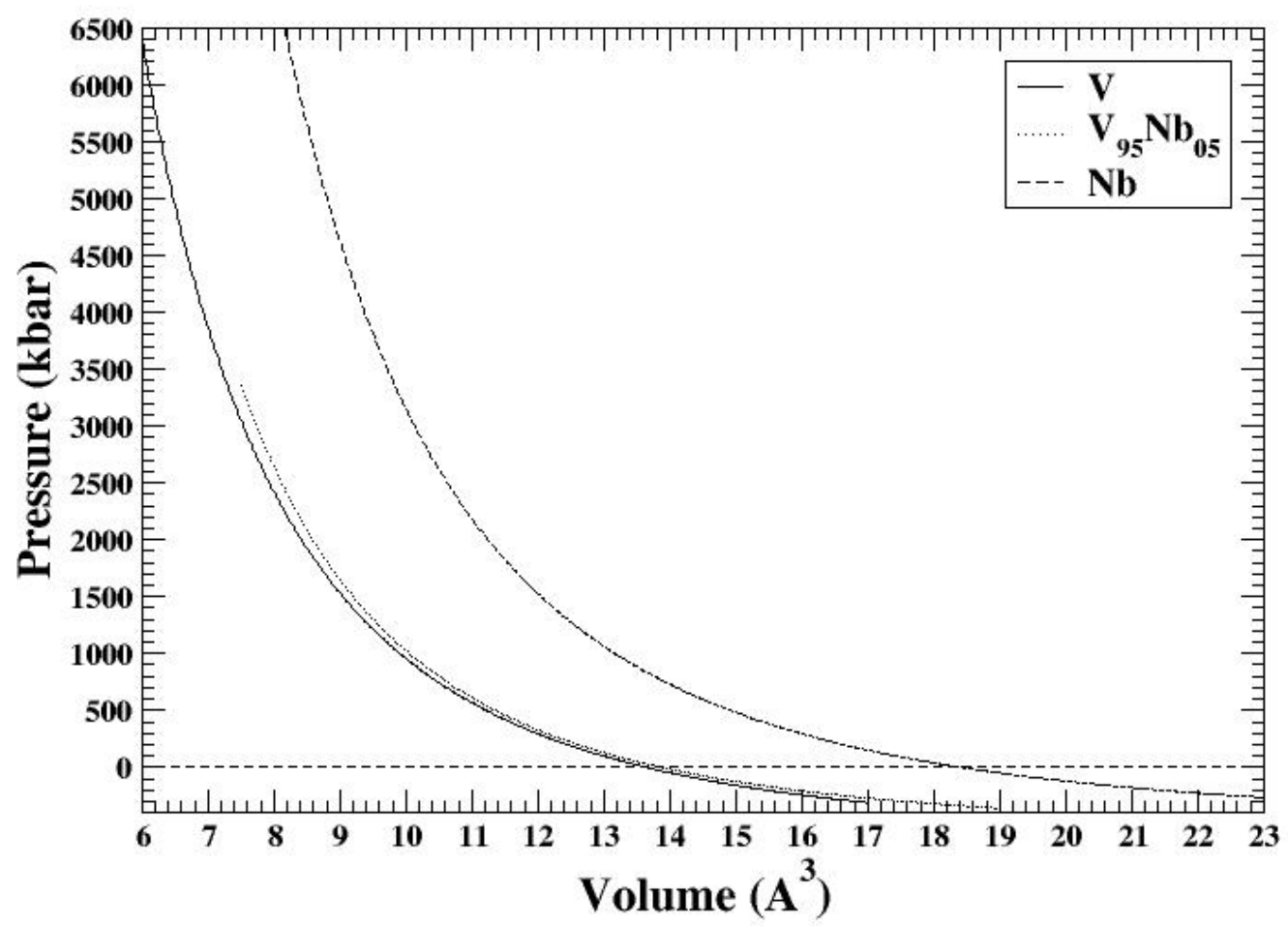

Figure 1. Equations of state of $\mathrm{V}, \mathrm{Nb}$, and the disordered $\mathrm{V}_{95} \mathrm{Nb}_{05}$ alloy. 


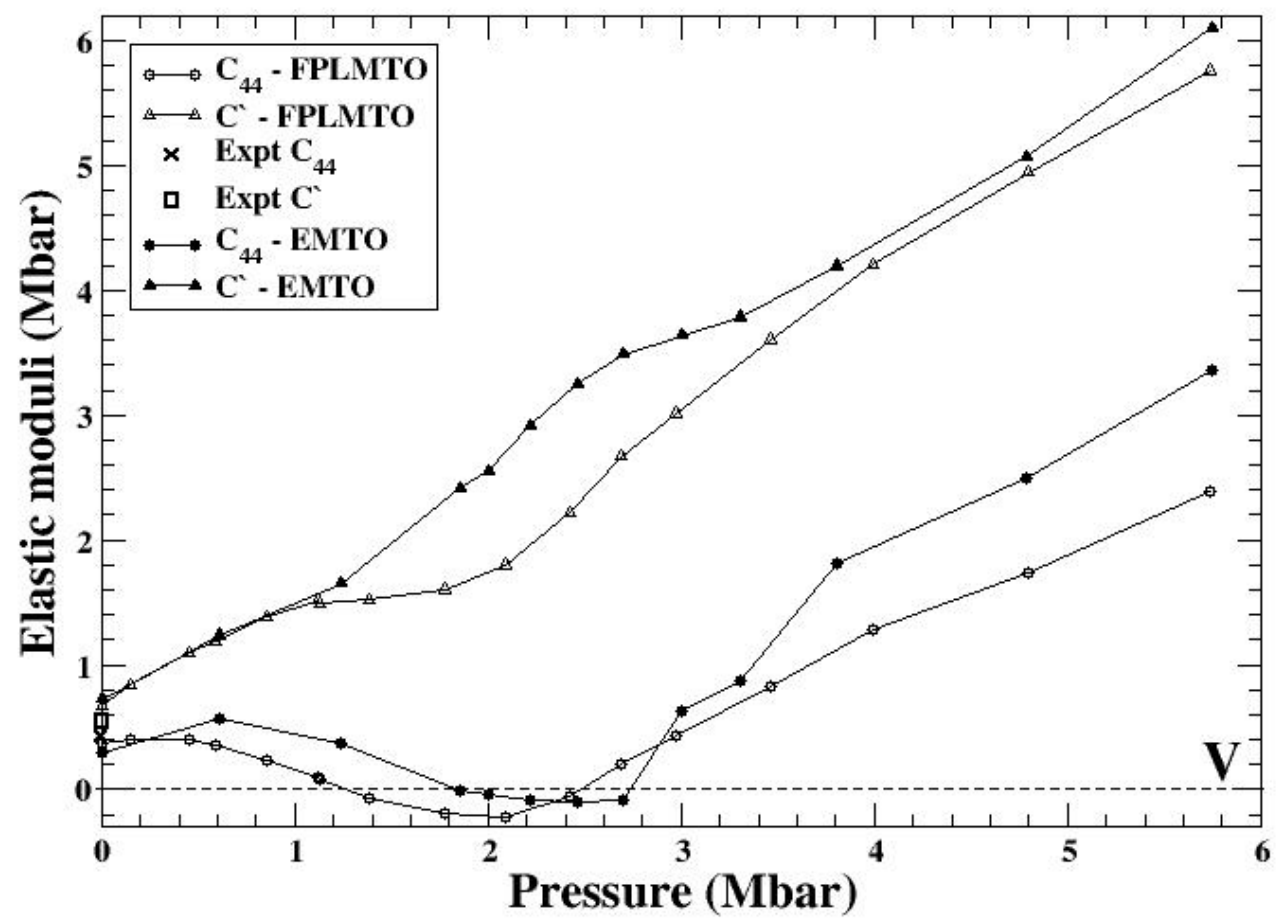

Figure 2. Pressure dependence of the shear elastic moduli of V. Experimental data at ambient pressure are according to Ref. [38]. 


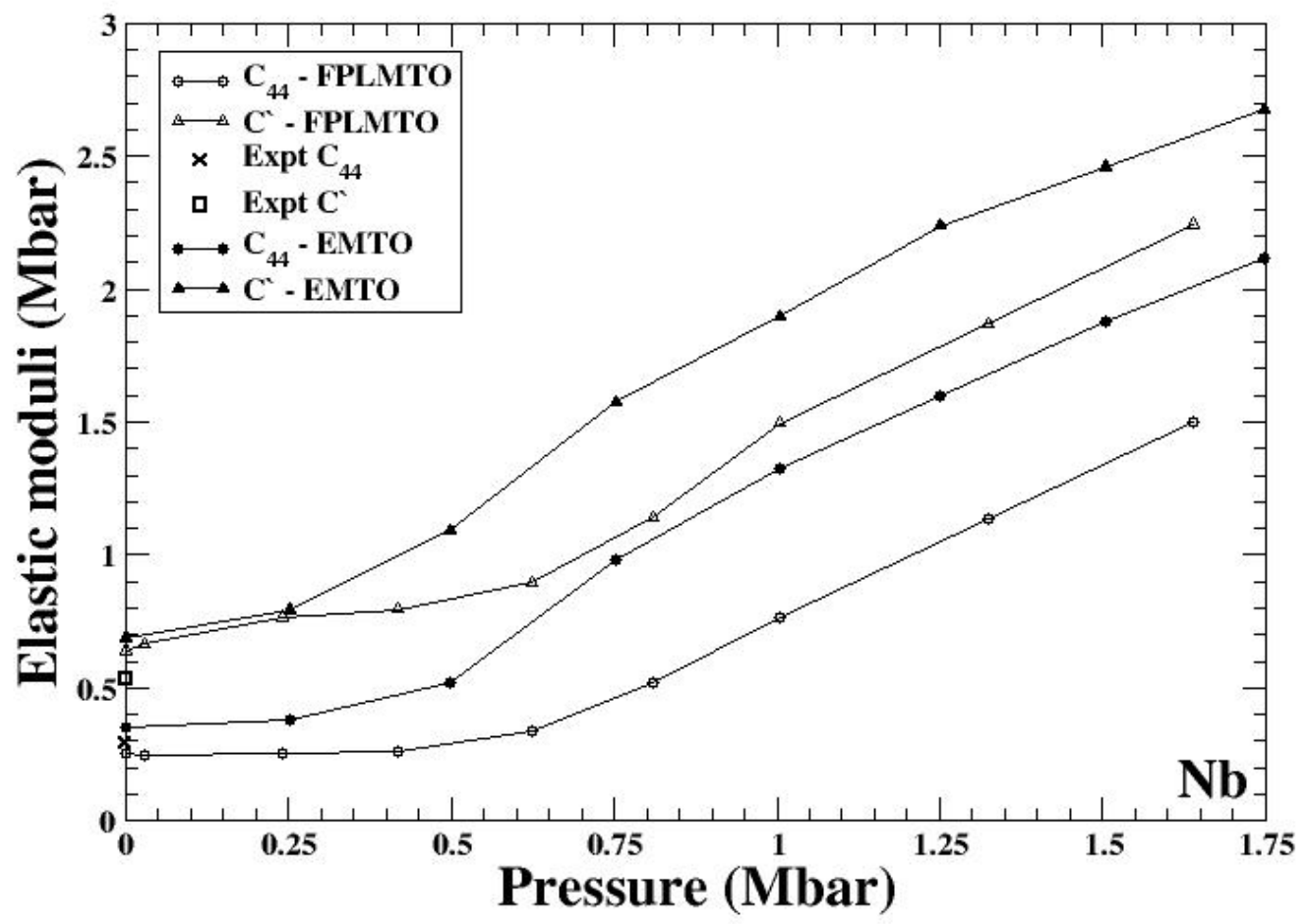

Figure 3. Pressure dependence of the shear elastic moduli of Nb. Experimental data at ambient pressure are according to Ref. [38]. 


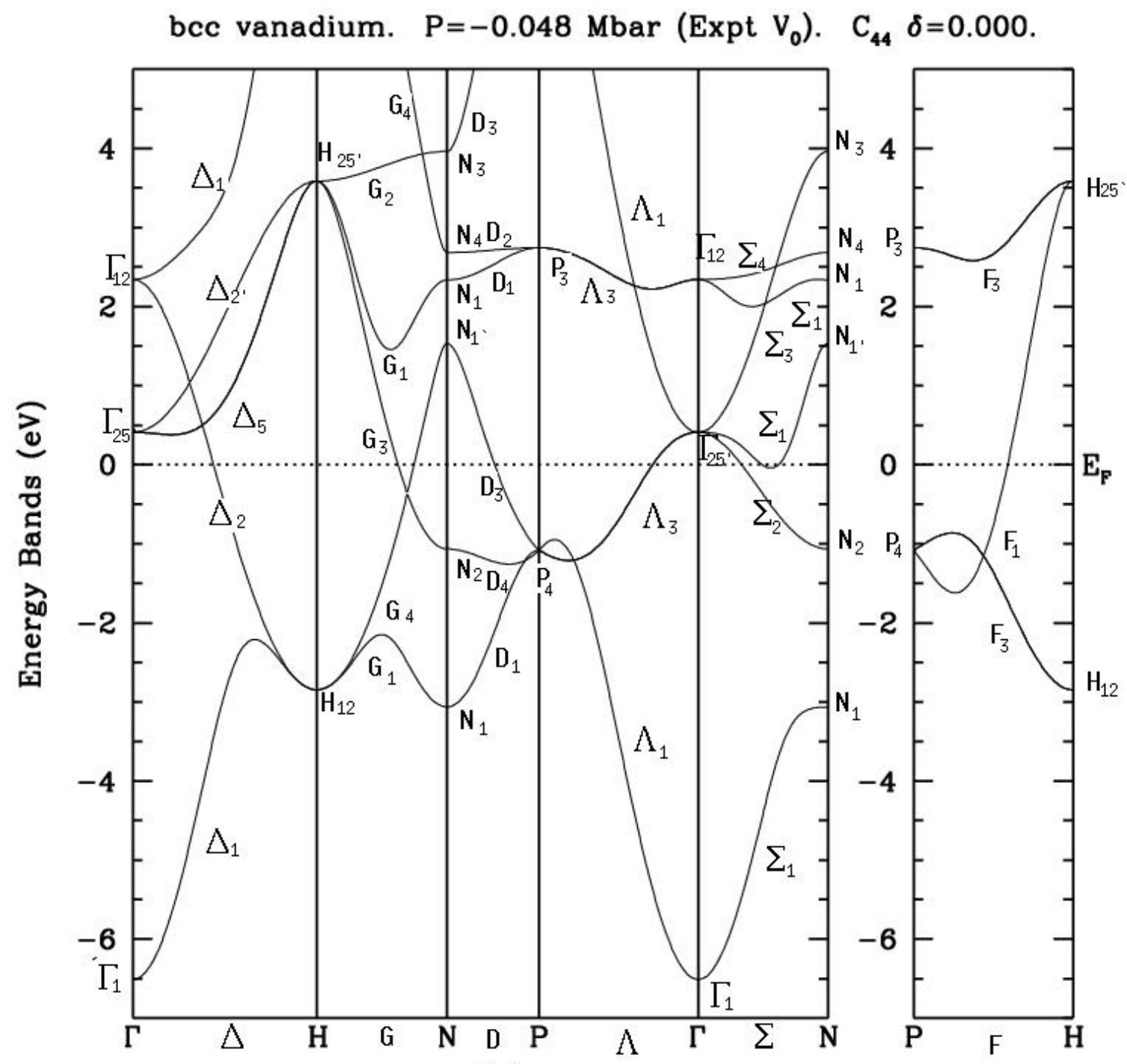

(a)

Figure 4a. Band energies of $\mathrm{V}$ at experimental equilibrium volume: undistorted. 


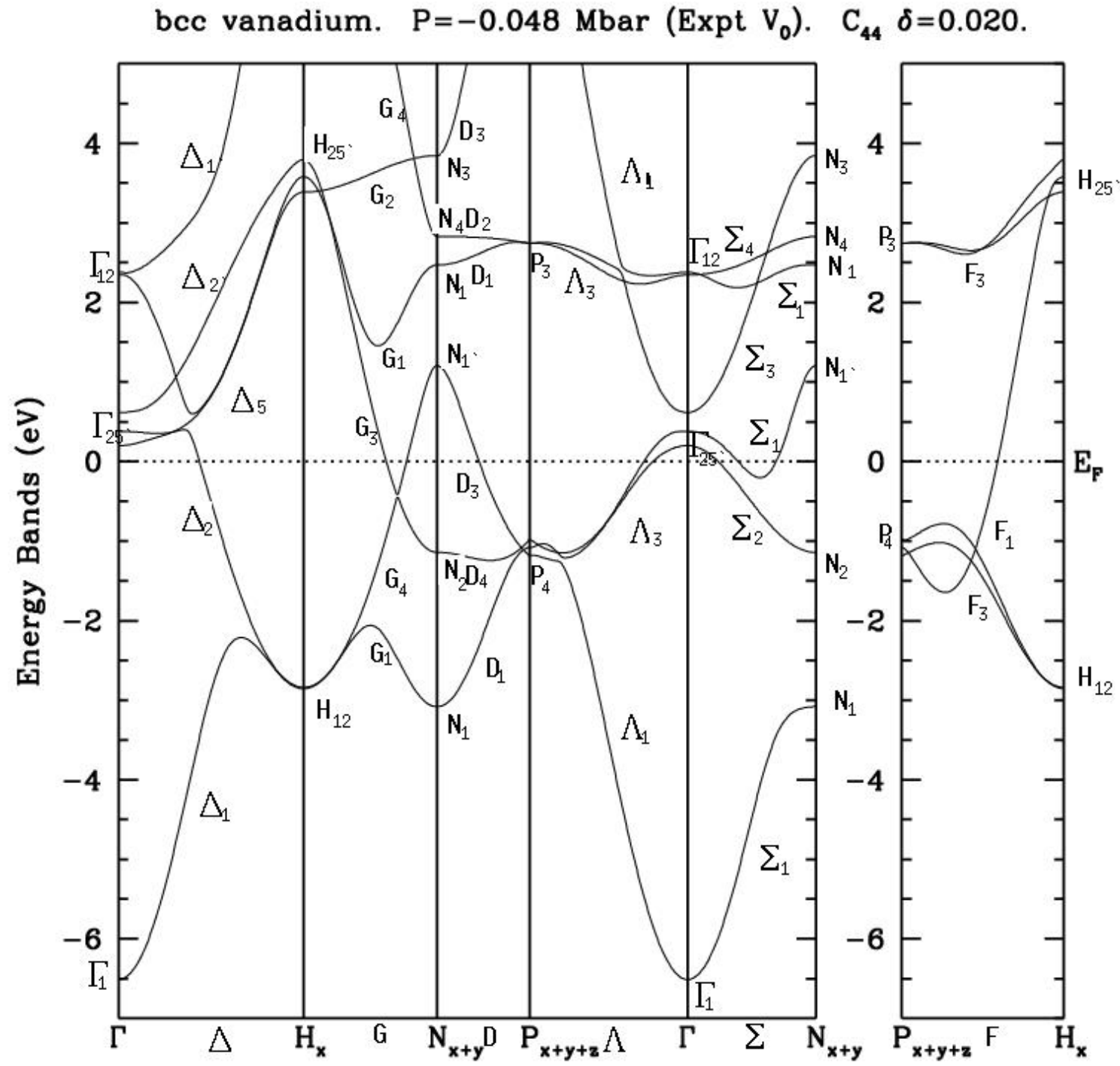

(b)

Figure $4 \mathrm{~b}$. Band energies of $\mathrm{V}$ at experimental equilibrium volume: distorted. 


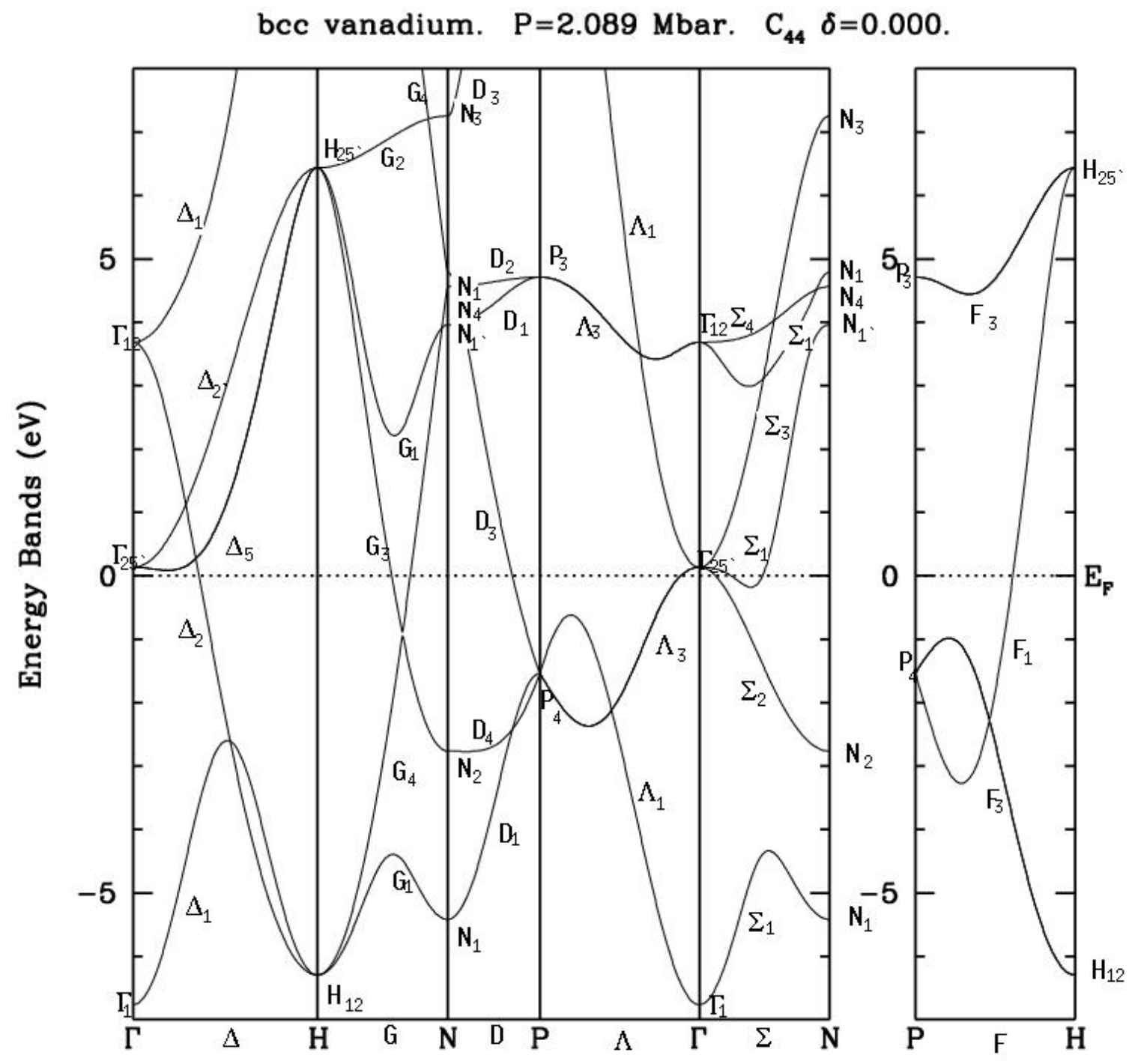

(a)

Figure 5a. Band energies of $\mathrm{V}$ at 2.089 Mbar: undistorted. 


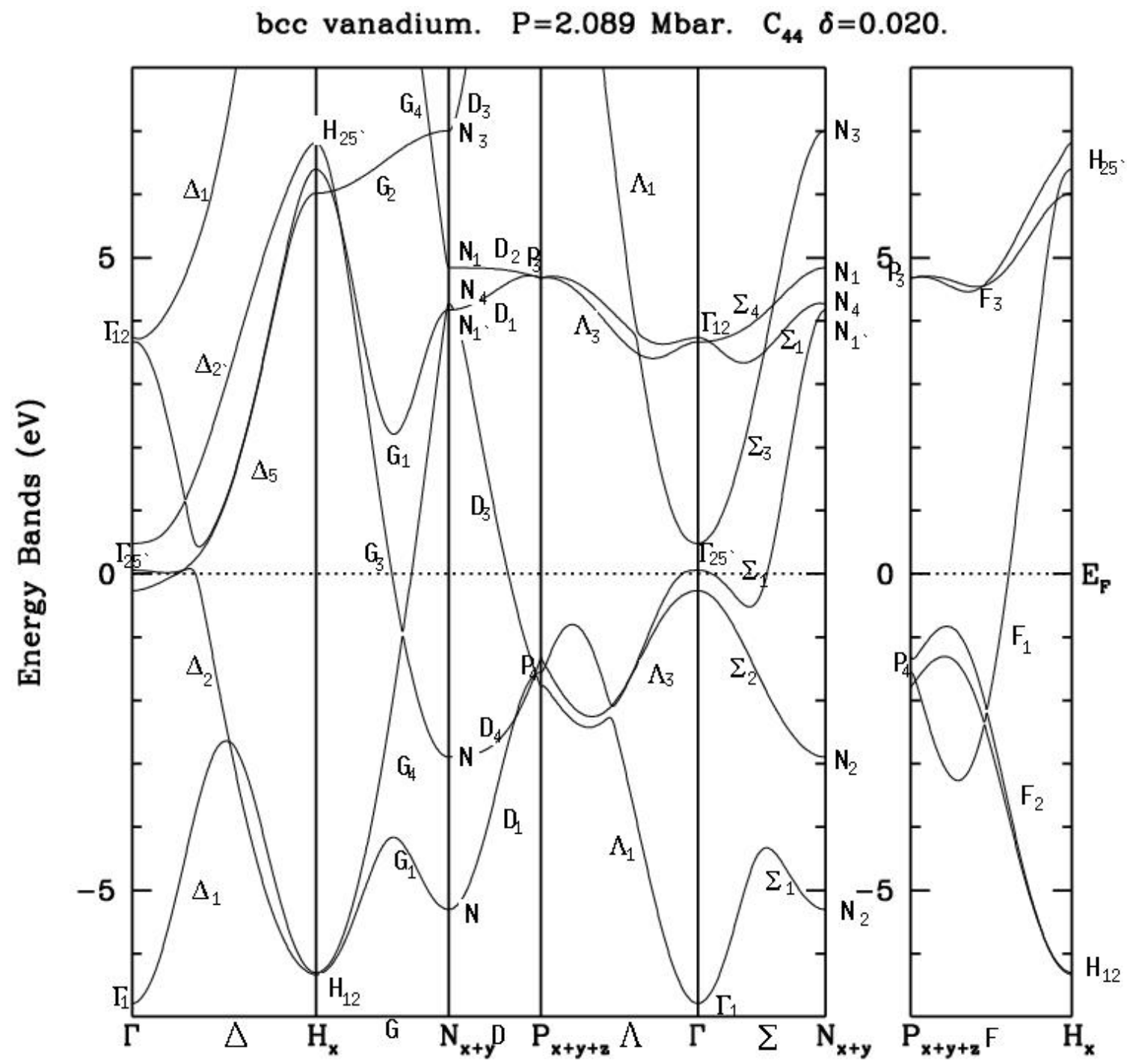

(b)

Figure 5b. Band energies of V at 2.089 Mbar: distorted. 


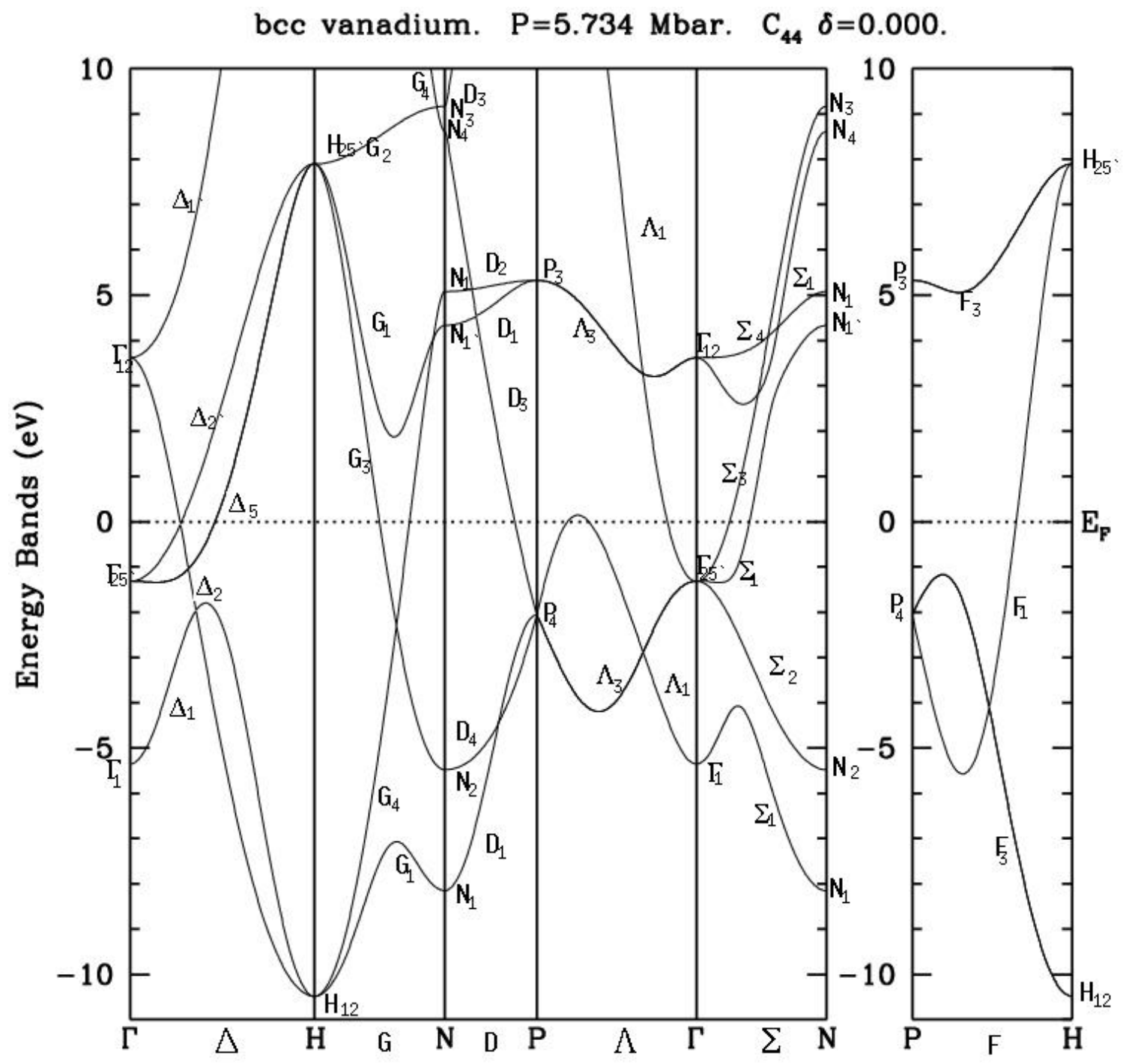

(a)

Figure 6a. Band energies of V at 5.734 Mbar: undistorted. 


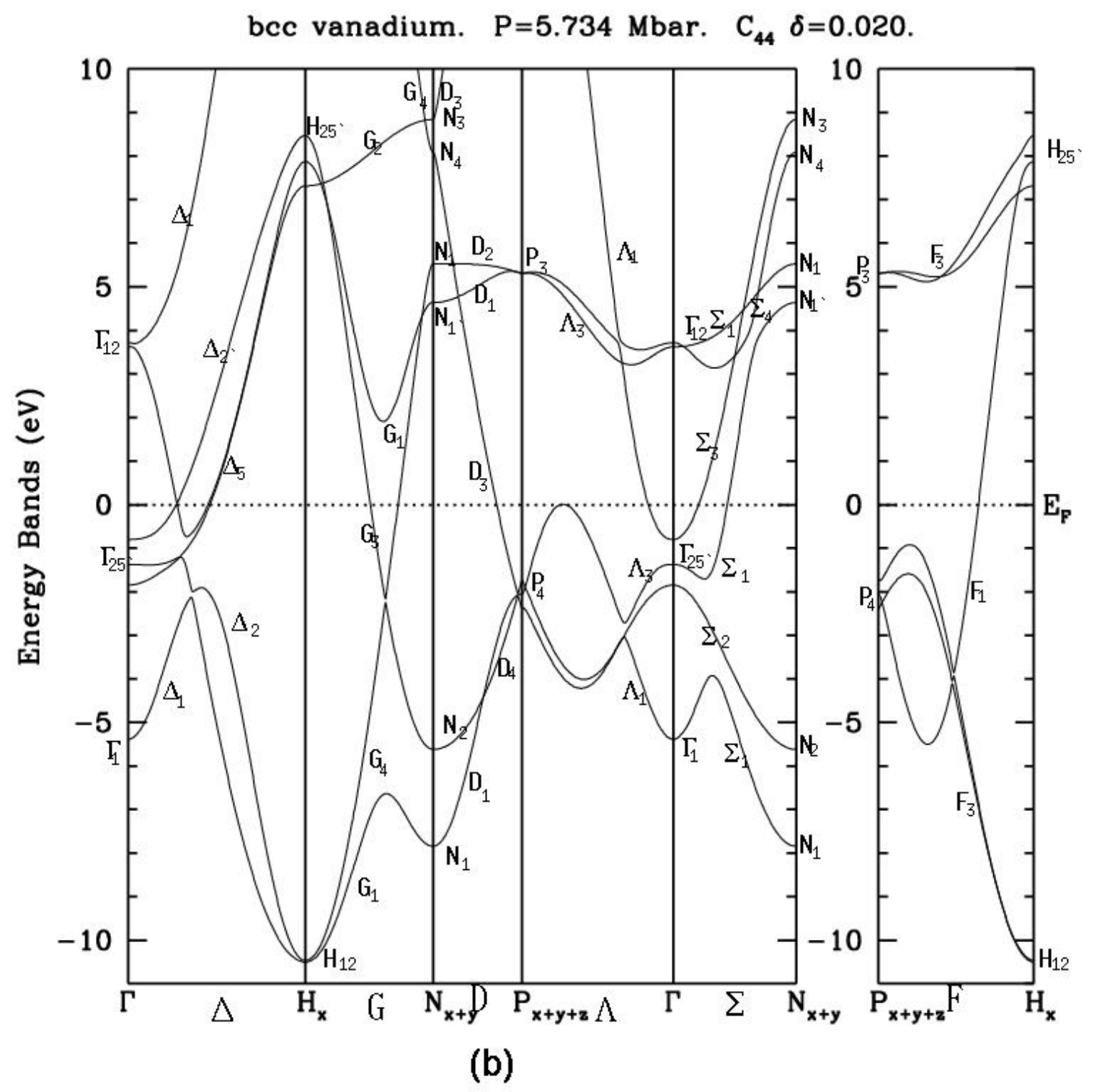

Figure 6b. Band energies of V at 5.734 Mbar: (b) distorted. 
(a)

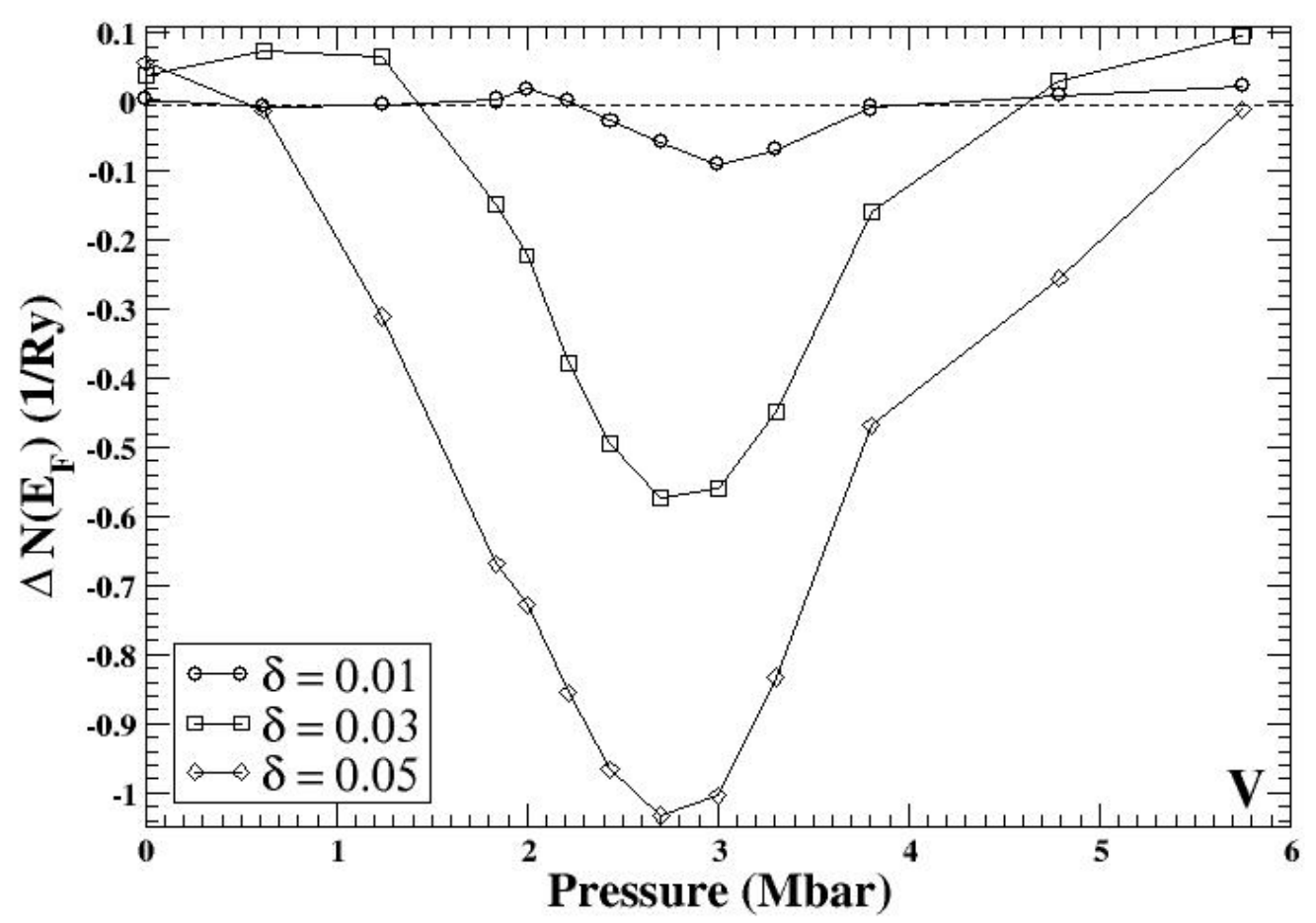

Figure 7a. The increment of the DOS at the Fermi level of V as a function of pressure. 
(b)

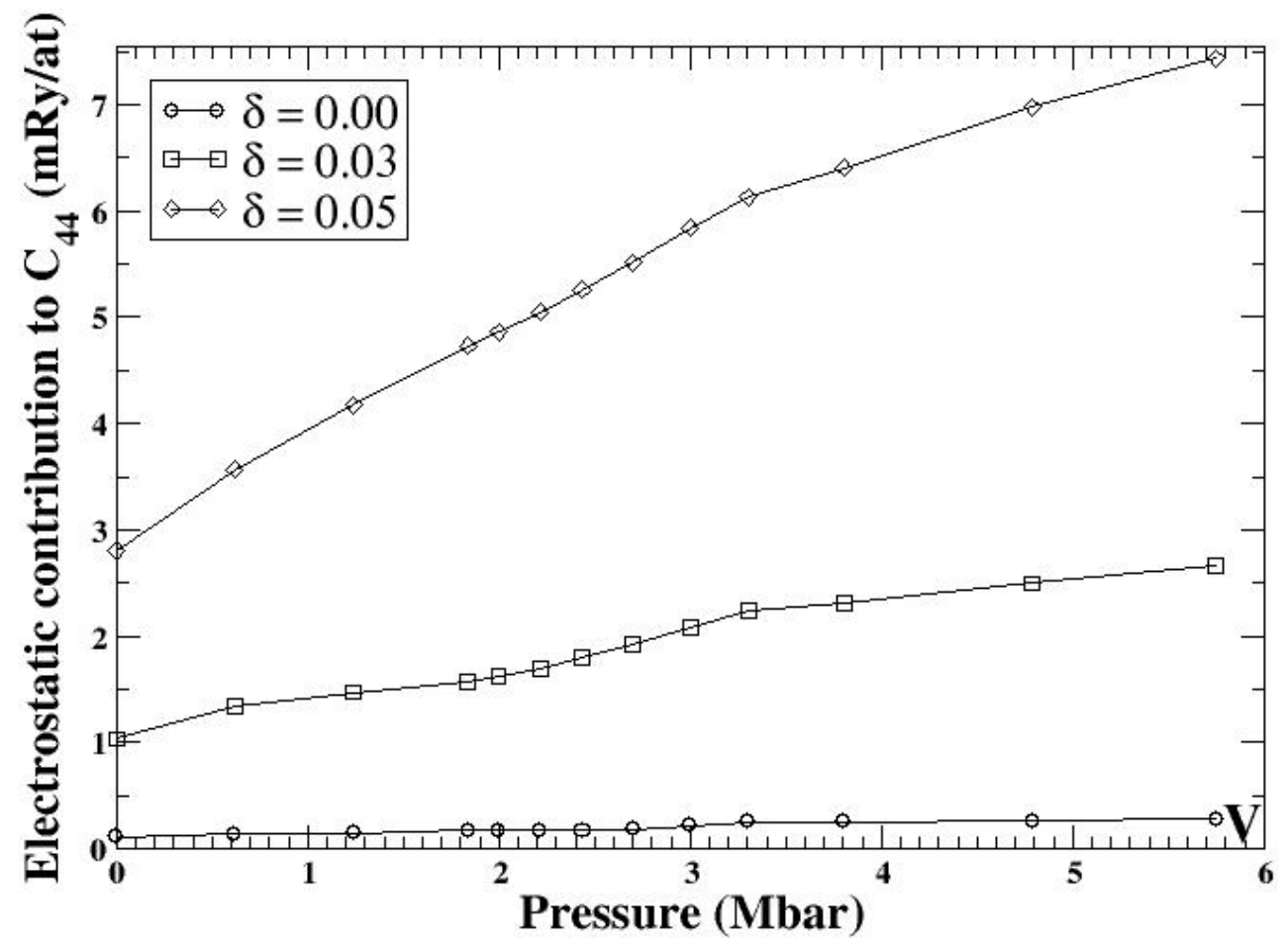

Figure $7 \mathrm{~b}$. The electrostatic contribution to the $\mathrm{C}_{44}$ elastic modulus of $\mathrm{V}$ as a function of pressure. 


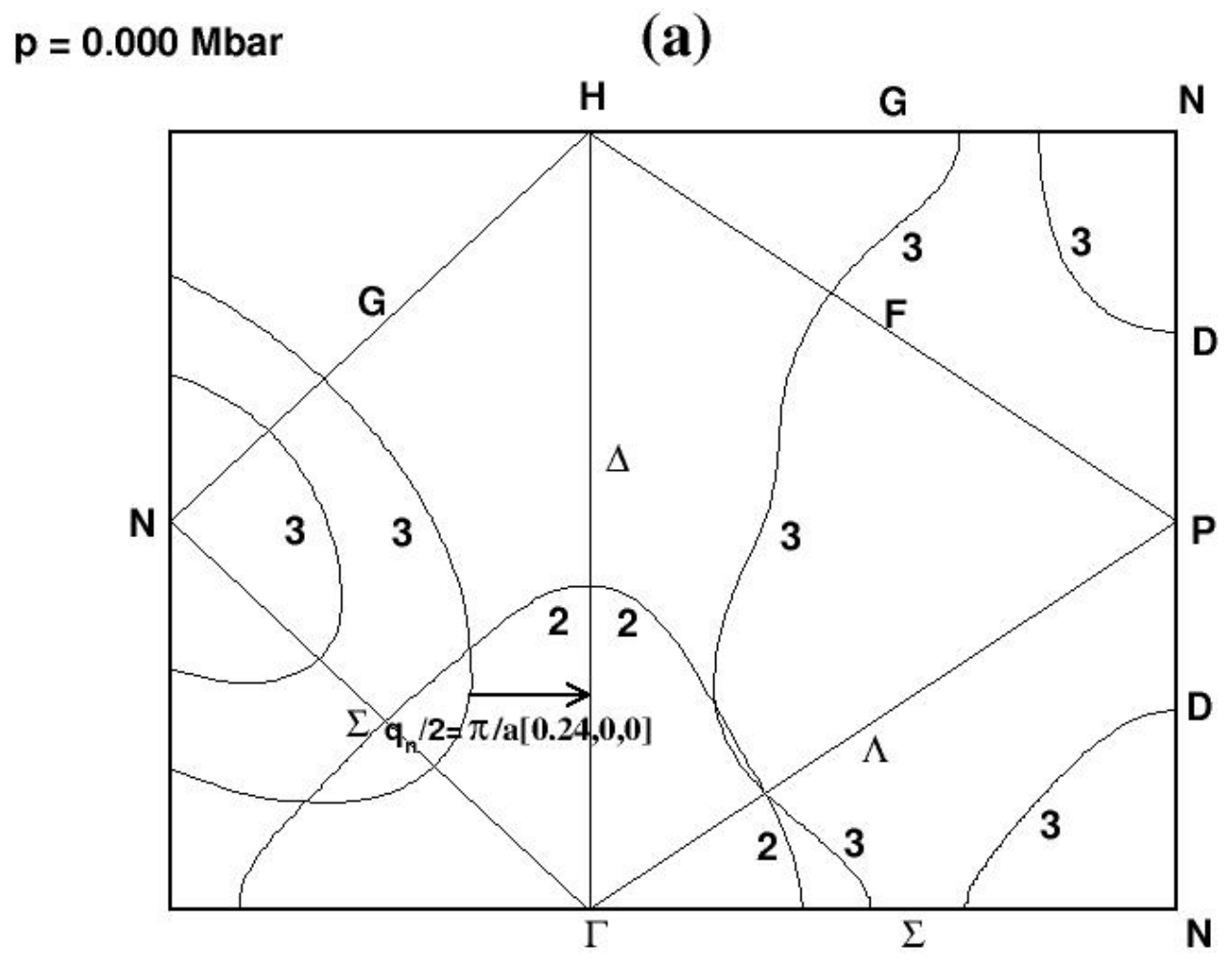

Figure 8a. Central $\{100\}$ and $\{110\}$ cross sections of the FS of V at ambient pressure. 


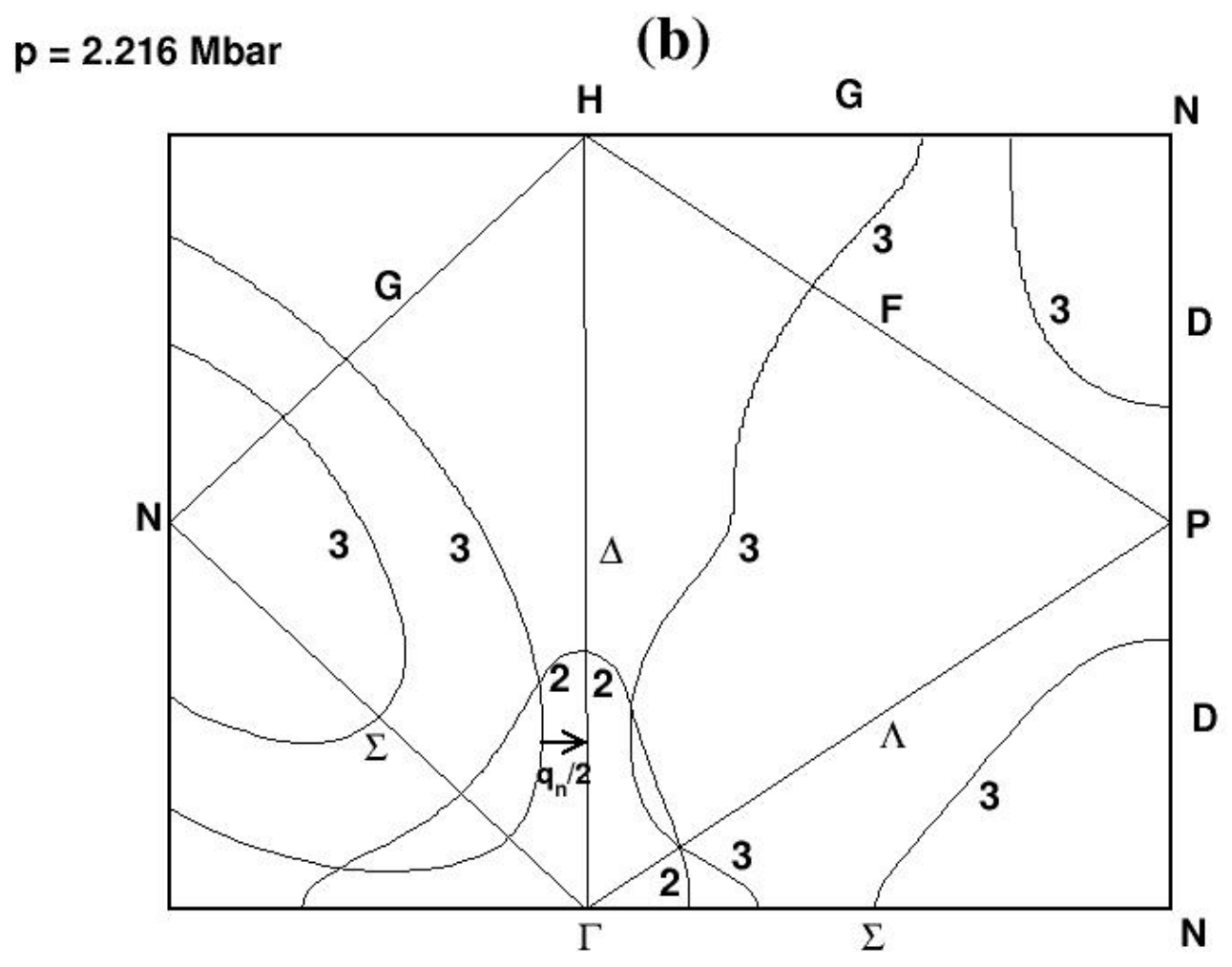

Figure $8 \mathrm{~b}$. Central $\{100\}$ and $\{110\}$ cross sections of the FS of V at $2.216 \mathrm{Mbar}$. 


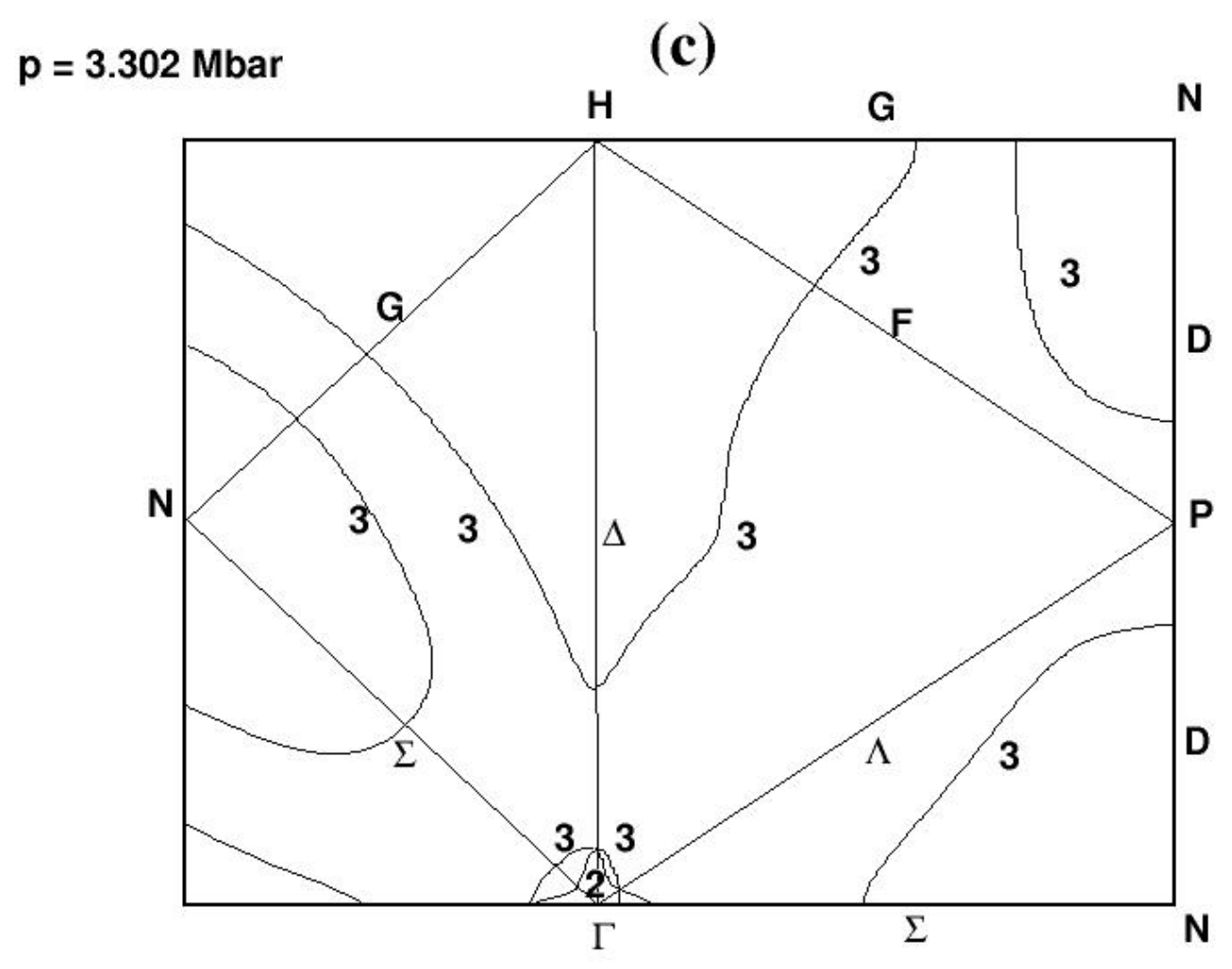

Figure 8c. Central $\{100\}$ and $\{110\}$ cross sections of the FS of V at 3.302 Mbar. 
(a)

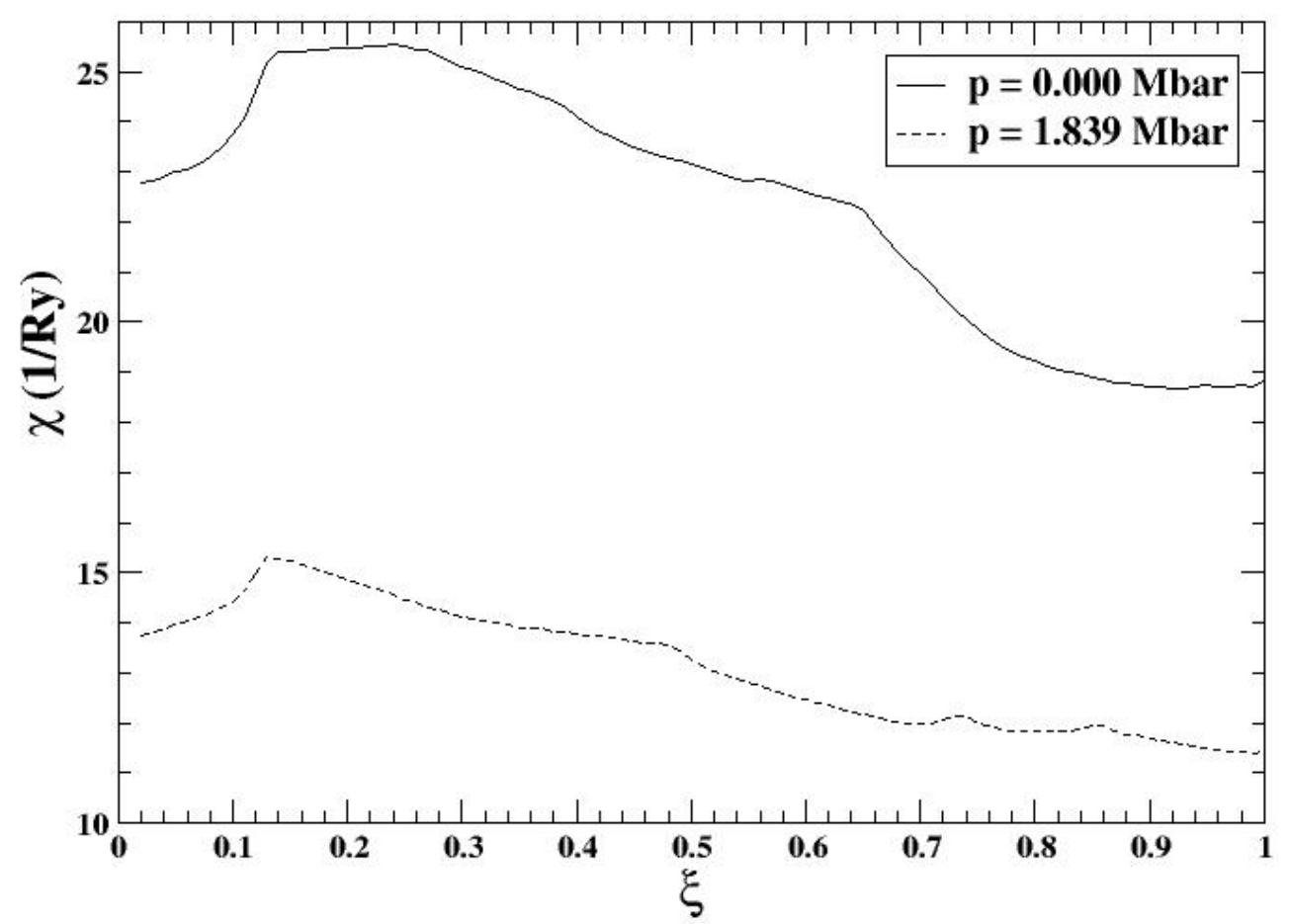

Figure 9a. Generalized total electron susceptibility of $\mathrm{V}$ along the $\Gamma-H$ ([100]) direction. 
(b)

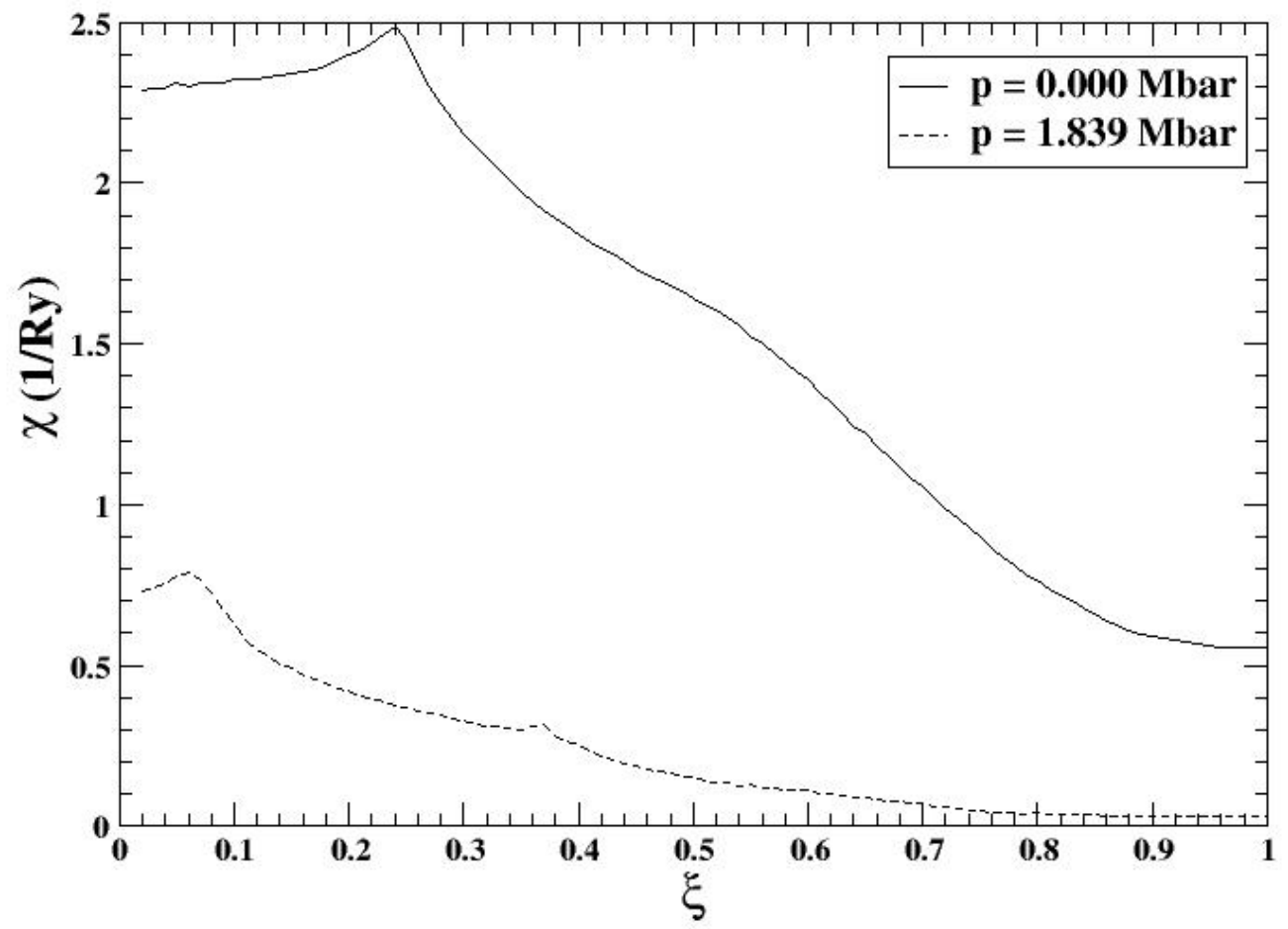

Figure $9 \mathrm{~b}$. Generalized partial ( $3^{\text {rd }} \rightarrow 3^{\text {rd }}$ intra-band transition) electron susceptibility of V along the $\Gamma-H([100])$ direction. 


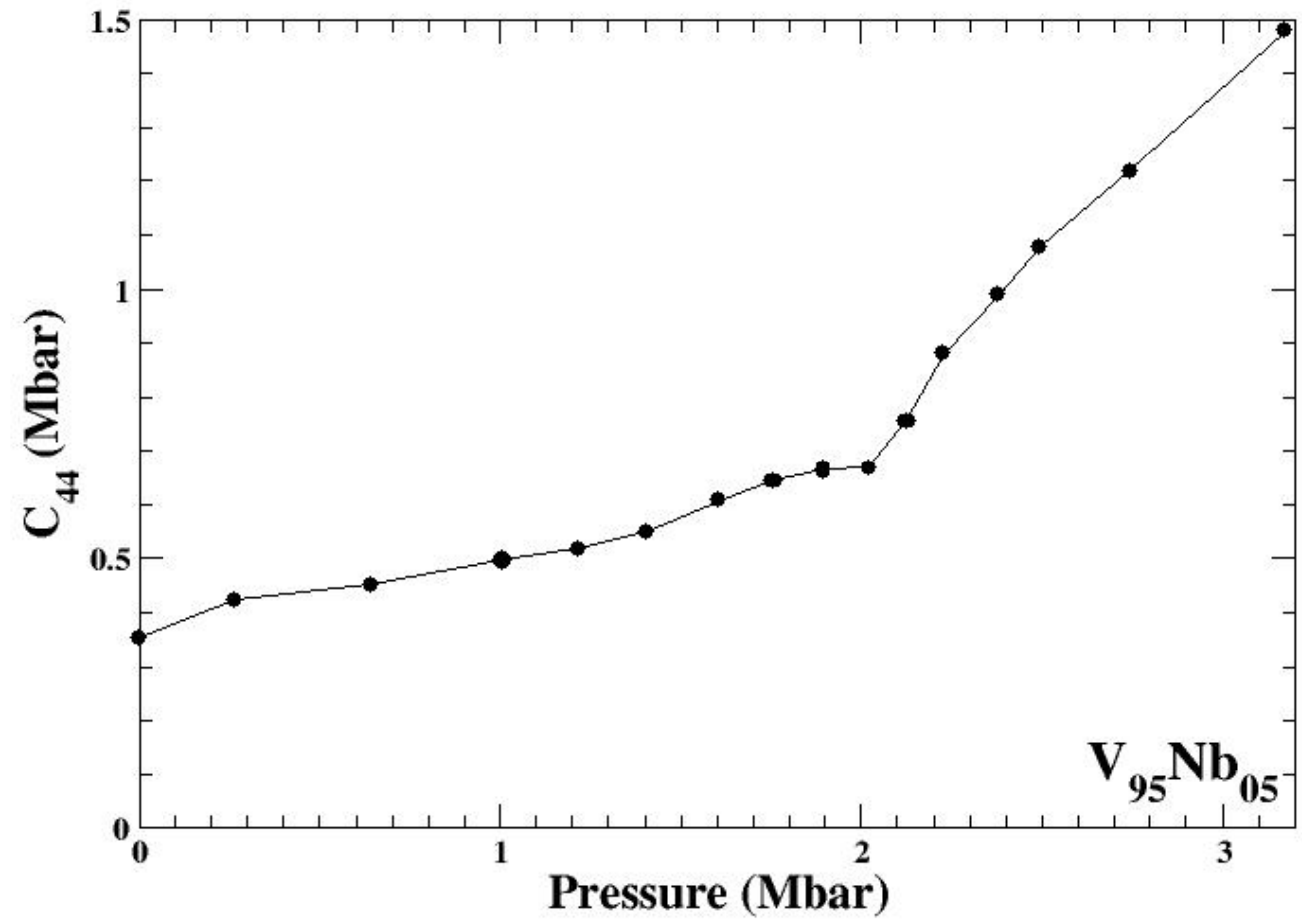

Figure 10. Pressure dependence of the shear elastic modulus $C_{44}$ of the disordered $\mathrm{V}_{95} \mathrm{Nb}_{05}$ alloy. 


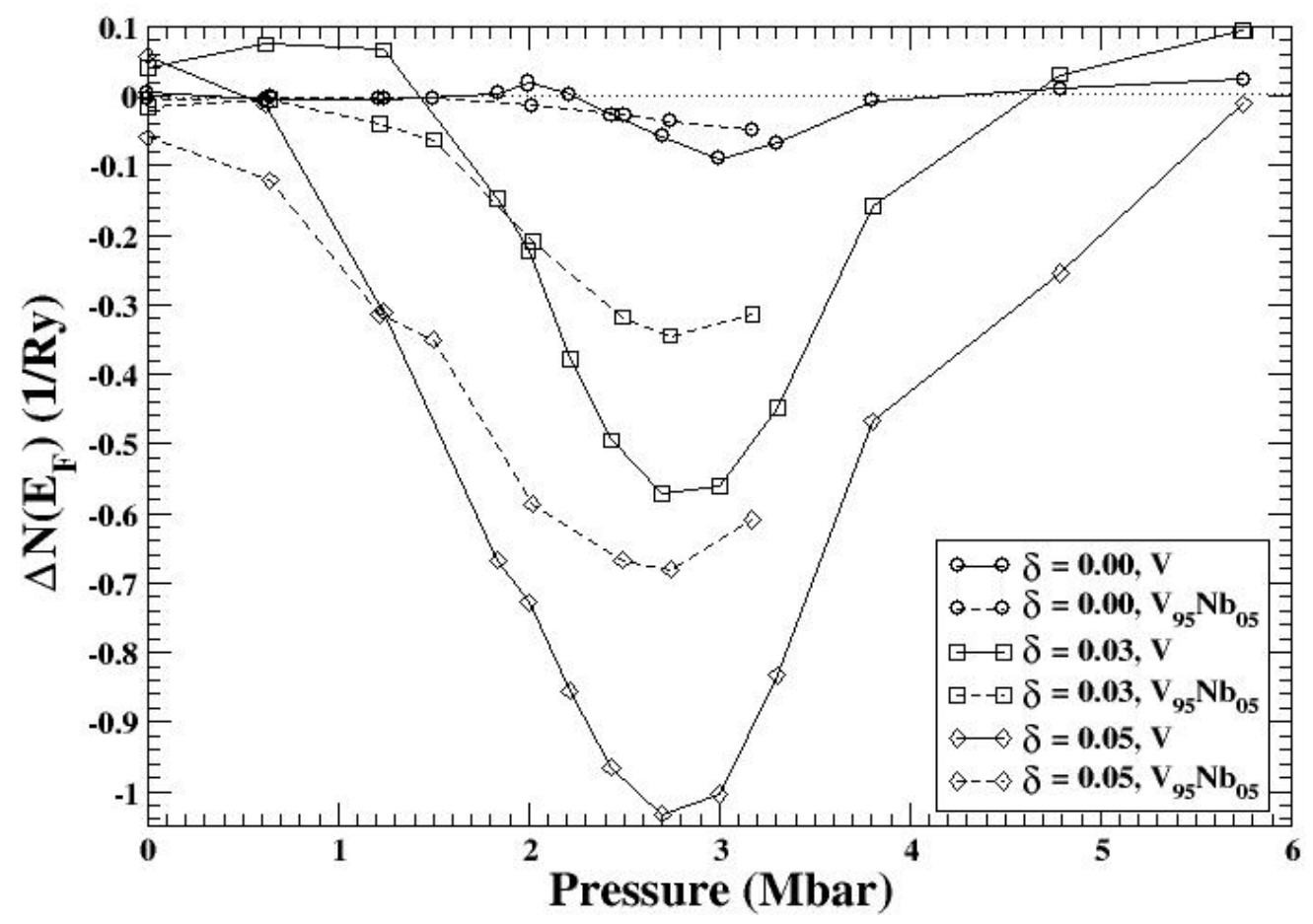

Figure 11. The increment DOS at the Fermi level of $\mathrm{V}$ and the disordered $\mathrm{V}_{95} \mathrm{Nb}_{05}$ alloy as a function of pressure. 